\title{
Forehead Flap in Coverage of Nasal Defects: Our Experience
}

\author{
Mohamed Elsayed Mohamed Mohamed ${ }^{*}{ }^{(0)}$, Ahmed Fathy Mohamed Eldehn ${ }^{2}$ \\ ${ }^{1}$ Department of Plastic Surgery, Ahmed Maher Teaching Hospital, Cairo, Egypt \\ ${ }^{2}$ Department of ENT, Kasr Al-Ainy Medical School, Cairo, Egypt \\ Email: ^Mghabn5777@yahoo.com, m.elsayed.tajmeel@gothi.cloud.gov.eg
}

How to cite this paper: Mohamed, M.E.M. and Eldehn, A.F.M. (2020) Forehead Flap in Coverage of Nasal Defects: Our Experience. Modern Plastic Surgery, 10, 1-8. https://doi.org/10.4236/mps.2020.101001

Received: October 19, 2019

Accepted: November 5, 2019

Published: November 8, 2019

Copyright $\odot 2020$ by author(s) and Scientific Research Publishing Inc. This work is licensed under the Creative Commons Attribution International License (CC BY 4.0).

http://creativecommons.org/licenses/by/4.0/

\begin{abstract}
Background: Coverage of post-traumatic or post-oncosurgical nasal defects is a very challenging procedure. Small nasal defects may be covered by skin grafts or small local flaps while larger nasal defects require more complex flap coverage techniques as using tissue expanders, prefabricated flaps or free flaps. The forehead flap has been used for centuries and remains a workhorse flap for reconstruction of large and complex nasal defects. Aim: evaluate the feasibility and versatility of forehead flap for resurfacing nasal defects. Materials and Methods: 12 patients underwent coverage of nasal defects after trauma or tumor excision using forehead flaps. All flaps needed a second stage for flap separation 3 weeks after the time of operation. The size of the harvested flap, the harvesting time, results of transferred flaps, patient satisfaction and flap-related complications were analyzed. Results: 12 patients ( 10 males and 2 females) underwent reconstruction of different nasal defects using 12 forehead flaps. The overall complications occurred in 2 patients. The remaining 10 patients showed no complications and passed an uneventful follow-up period. 7 Patients were very satisfied, 4 were satisfied and only one patient was not satisfied as she was 23 years old young female and was subjected to human bite. Follow-up periods ranged from 6 to 12 months. Conclusion: Nasal defects can successfully be managed with the forehead flap. Probably, the flap provides the best result due to the good matching of the skin in terms of color, texture, and thickness. Also, despite the increasing use of free flaps, the forehead flap is still a valid and safe option for nasal defects coverage that allows good aesthetics and functional outcomes.
\end{abstract}

\section{Keywords}

Nasal Defects, Forehead Flap, Flap Coverage, Nasal Reconstruction 


\section{Introduction}

Nose is frequently vulnerable to trauma and skin cancers due to its prominent position in the center of the face [1].

The technique of nasal reconstruction originated almost 3000 years ago in India, where nasal amputation was a common way of social punishment for various crimes. This required methods of total or subtotal nasal reconstruction [2].

In the late 18th century, Carpue found a description of this Indian technique, which gave rise to the modern era of nasal resurfacing with the use of a pedicled forehead flap. His basic technique was the start-up for modern nasal reconstruction for the next century. These techniques were later modified and popularized by other surgical pioneers [3].

Burget \& Menick introduced the concept of aesthetic units of the nose, claiming that the incisions should be located at the limits of these aesthetic units. If it is necessary to remove more than one third of a unit, then the whole aesthetic unit must be completely removed and reconstructed [4].

There are several options to cover nasal defects. Among the available methods, the median forehead flap has been used for centuries and remains a workhorse flap for reconstruction of major and complex nasal defects [5] [6].

The forehead flap has undergone a high level of development and improvement, making it the optimal choice for large nasal defects. It is traditionally limited to use for nasal defects that are too large to repair with other smaller local flaps or full-thickness or composite grafts. It is considered the gold standard for all nasal reconstruction. Additional principles that have improved outcomes include maintaining an axial pattern whenever possible, utilizing the ipsilateral pedicle to the defect and extension of the flap at right angles across the forehead for additional reach when necessary [7] [8].

This study aims to demonstrate the versatility of the flap in the forehead flap in nasal defects coverage, which provides adequate contour and good aesthetic outcome.

\section{Material and Methods}

From July 2015 to April 2019, patients who suffered from nasal trauma with skin loss and malignancy affecting nasal skin were admitted to undergo nasal resurfacing using the forehead flap. During this period 12 patients agreed to cover their defects using this method. Preoperative preparation was done for all patients, and consent was taken to use this flap for reconstruction, while other patients who did not agree were treated with other reconstructive methods.

Template was done for each case intraoperatively. The pedicle was located about $2 \mathrm{~cm}$ lateral to the midline near the medial eyebrow. The base of the flap was designed $2.5 \mathrm{~cm}$ wide to include the pedicle. Flap was elevated from distal to proximal direction. Dissection was done in subgaleal plane distally then near the pedicle, dissection was done in subperiosteal plane to include the vessel in the flap. Flap donor site was closed directly or using split thickness skin graft. 
All flaps needed a second stage for flap separation 3 weeks after the time of operation. The size of the harvested flap, the harvesting time, results of transferred flaps, patient satisfaction and flap-related complications were analyzed.

Severe complications were defined as more than one-fourth to the total loss of the flap. Moderate complications were defined as loss of less than one-fourth of the entire flap. A dehiscence or hematoma leading to impairment of wound healing was defined as a minor complication. Patient satisfaction was defined as very satisfied (VS), satisfied (S) and not satisfied (NS).

\section{Results}

12 patients ( 10 males and 2 females) underwent reconstruction of different nasal defects using 12 median forehead flaps. The nasal defects involved the nasal side walls, dorsum of the nose, nasal tip and alar nose areas caused by malignancy, trauma, burn and bites due to human or animal origin. Patient data are summarized in Table 1.

The average times of flap harvest were between 30 minutes to 75 minutes according to the experience of the surgeon and his hand craft. Mean flap sizes ranged from $(2 \mathrm{~cm} \times 10 \mathrm{~cm})$ to $(3 \mathrm{~cm} \times 12 \mathrm{~cm})$.

The overall Complications occurred in 2 patients ( 2 minor complications) represented $16 \%$ in the form of distal flap wound dehiscence, as shown in Table 2. The two cases underwent conservative treatment till complete healing of the wound dehiscence. The remaining patients representing $84 \%$ of total number showed no complications and passed an uneventful follow-up period. Characteristics of the flaps transferred are shown in Table 2.

Patient satisfaction is shown in Table 3:

Table 1. Demographic data of the patients with forehead flap nasal reconstruction.

\begin{tabular}{ccccc}
\hline Patient & Sex & Age & Diagnosis & Area Reconstructed \\
\hline 1 & M & 65 & Tumor & Side Wall \\
2 & F & 23 & Bite (Human) & Tip + Ala \\
3 & M & 63 & Tumor & Side Wall \\
4 & M & 69 & Tumor & Side Wall + Ala \\
5 & M & 70 & Tumor & Side Wall + Dorsum \\
6 & F & 22 & Trauma & Side Wall + Tip \\
7 & M & 33 & Trauma & Side Wall + Dorsum \\
8 & M & 44 & Trauma & Side Wall + Tip \\
9 & F & 68 & Tumor & Side Wall Wall + Ala \\
10 & M & 66 & Tumor & Tip + Ala \\
11 & M & 33 & Bite (Animal) & Side Wall + Dorsum \\
12 & F & 55 & Tumor &
\end{tabular}


Table 2. Forehead flap characteristics and complications of flap reconstruction.

\begin{tabular}{cc}
\hline Estimated Flap Dimensions $(\mathrm{cm} \times \mathrm{cm})$ & $2 \mathrm{~cm} \times 10 \mathrm{~cm}$ to $3 \mathrm{~cm} \times 12 \mathrm{~cm}$ \\
\hline Estimated Harvest Time (min) (mean) & $30 \mathrm{~min}$ to $90 \mathrm{~min}$ \\
Severe Complications & - \\
Moderate Complications & - \\
Minor Complications & 2 \\
\hline
\end{tabular}

Table 3. Patient satisfaction after forehead flap separation (the second stage).

\begin{tabular}{ccccccccccccc}
\hline Patient & $\mathbf{1}$ & $\mathbf{2}$ & $\mathbf{3}$ & $\mathbf{4}$ & $\mathbf{5}$ & $\mathbf{6}$ & $\mathbf{7}$ & $\mathbf{8}$ & $\mathbf{9}$ & $\mathbf{1 0}$ & $\mathbf{1 1}$ & $\mathbf{1 2}$ \\
\hline Satisfaction & VS & NS & VS & S & VS & VS & S & VS & VS & S & VS & S \\
\hline
\end{tabular}

7 patients were very satisfied, 4 patients were satisfied and only one patient was not satisfied. She was 23 years old and was subjected to human bite by one of her coworkers. This represented about (8\%) of all cases operated. Follow-up periods ranged from 6 to 12 months.

\section{Discussion}

The forehead is known as the ideal donor site for nasal resurfacing due to its skin quality, size, and vascularity. The forehead is perfused inferiorly from the supratrochlear and supraorbital vessels and superiorly and laterally from the superficial temporal, post auricular, and occipital vessels [8].

During our study, 12 defects of the nose were reconstructed using forehead flap with its variants. All patients accomplished successful nasal resurfacing although 2 patients representing (18\%) suffered from minor complication in the form of dehiscence along the distal 2-mm border of the skin paddle. This area was treated by secondary sutures and re-epithelialized uneventfully.

No flaps suffered full-thickness necrosis or congestion that required intervention. This conforms to the results of Stephen and park, who repaired 10 patients with single-stage forehead flap reconstruction and only 1 patient suffered epidermolysis while the rest of patients passed uneventful postoperative period [9].

We also agree with Millard, who stated that for nasal reconstructions, the midline forehead skin flap can serve as a cover for any nasal reconstruction from severe tip and alar loss to a total nasal defect. Using this flap, aesthetic and functional reconstruction can be achieved by creating a nose that blends well with the face [10].

It appears that in the recent history of head and neck reconstruction, pedicled and free flaps have concurred for the same indications and that their use, in some cases, can be mutually exclusive [1].

Still, free flaps are considered the reference standard for many cases of head and neck reconstruction; however, a significant body of data has been increasing slowly but steadily in which pedicled flaps have been used in comparable settings. In many instances, pedicled regional or micro-vascular soft tissue flaps compete for 
the same indication, each technique with its advantages and disadvantages [11].

Different case series with each other conclude that pedicled and free flaps are equally reliable [1] [8].

Out of the 12 patients underwent nasal defect coverage only one patient was not satisfied with the result. This might be due to the cause of nasal defect and her young age ( 23 years old). The patient had no time to experience the nasal defect which was due to a bite by one of her coworkers.

We need larger numbers of patients to be included in wider scales of studies to evaluate the versatility and feasibility of the flap for nasal reconstruction.

The use of forehead flap can successfully cover different nasal defects with good results and minimal or no complications. Even in the presence of free flap reconstructions, the regional flaps still have a great rule and still compete with the free flaps in coverage of the nasal defects with a good aesthetic outcome.

\section{Conclusion}

Nasal defects can successfully be managed with the forehead flap with its variants. Probably, the flap provides the best result due to the good matching of the skin in terms of color, texture, and thickness. Also, despite the increasing use of free flaps, the forehead flap is still a valid and safe option for nasal defects coverage that allows good aesthetics and functional outcomes.

\section{Ethical Approval}

- The manuscript has not been submitted to any other journals for simultaneous consideration.

- The manuscript has not been published previously (partly or in full).

- No data have been fabricated or manipulated (including images) to support our conclusions

- No data, text, or theories by others are presented as if they were our own.

- Consent to submit has been received from all co-authors.

- Authors whose names appear on the submission have contributed sufficiently to the scientific work and therefore share collective responsibility and accountability for the results.

- All procedures performed in this study involving patients were in accordance with the ethical standards of our research committee.

- Informed consent was obtained from the patient herself for surgical intervention and also for publication of photos.

\section{Conflicts of Interest}

The authors declare no conflicts of interest regarding the publication of this paper.

\section{References}

[1] Rohrich, R.J., Griffin, J.R., Ansari, M., Beran, S.J. and Potter, J.K. (2004) Nasal Re- 
construction-Beyond Aesthetic Subunits: A 15-Year Review of 1334 Cases. Plastic and Reconstructive Surgery, 114, 1405-1416.

https://doi.org/10.1097/01.PRS.0000138596.57393.05

[2] Converse, J.M. (1977) Corrective and Reconstructive Surgery of the Nose. In: Converse, J.M., Ed., Reconstructive Plastic Surgery (2nd Ed.), Saunders, Philadelphia.

[3] Rohrich, R.J., Barton, F.E. and Hollier, L. (1997) Nasal Reconstruction. In: Aston, S.J., Beasley, R.W., Thorne, C.H.M., Eds., Grabb and Smith's Plastic Surgery (5th Ed.), Lippincott-Raven, Philadelphia, 513-529.

[4] Brenner, M.J. and Moyer, J.S. (2017) Skin and Composite Grafting Techniques in Facial Reconstruction for Skin Cancer. Facial Plastic Surgery Clinics of North America, 25, 347-363. https://doi.org/10.1016/j.fsc.2017.03.007

[5] Mukta, V. and Brijesh, M. (2018) Complex Nasal Reconstruction Using Extended Paramedian Forehead Flap: A Case Report. Cancer Biology and Therapeutic Oncology, 2, 2.

[6] Cintra, H.P.L. Ama, A.B., Holanda, T., et al. (2013) Use of Mid-Forehead Flap in Nasal Reconstruction. Revista Brasileira de Cirurgia Plástica, 28, 212-217. https://doi.org/10.1590/S1983-51752013000200007

[7] Moghadam, M.A. and Moghadam, S.A. (2017) Use of Forehead Flap for Nasal Tip Reconstruction after Traumatic Nasal Amputation (A Case Report). World Journal of Plastic Surgery, 6, 361-364.

[8] Menick, F.J. (2010) Nasal Reconstruction. Plastic and Reconstructive Surgery, 125, $1-14$.

[9] Stephen, S. and Park, M.D. (2002) The Single-Stage Forehead Flap in Nasal Reconstruction an Alternative with Advantages. Archives of Facial Plastic Surgery, 4, $32-36$.

[10] Millard, D.R. (2009) Midline Forehead Skin Flap. In: Berish, S., Ed., Grabb’s Encyclopedia of Flaps, Lippincott Williams \& Wilkins, Philadelphia, 99-100.

[11] Giacomo, C., Karim, T., Alessandro, B., Fabiana, A., Matteo, C., Marco, M. and Dimitri, R. (2015) Regional Flaps in Head and Neck Reconstruction: A Reappraisal Journal of Oral and Maxillofacial Surgery, 73, e1-e571. https://doi.org/10.1016/j.joms.2014.10.021 


\section{Cases Figures Legends}

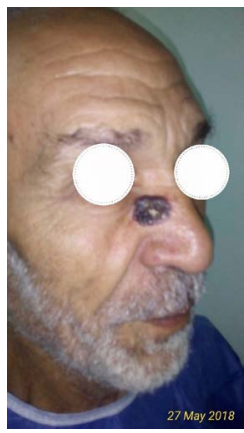

(a)

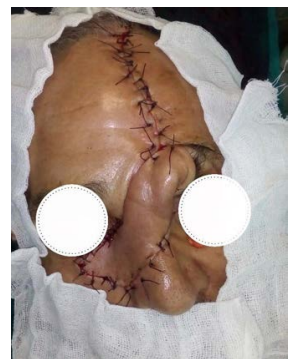

(c)

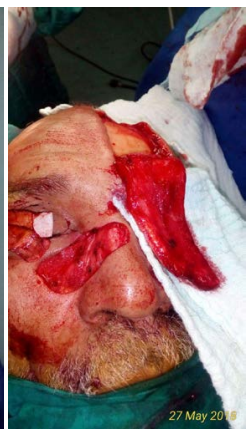

(b)

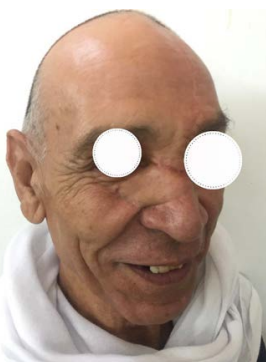

(d)

Case 1. A 68-year-old male with basal cell carcinoma of the right upper medial check and lateral nasal wall. (a) Pre-operative Antero-lateral view of the face showing the tumor. (b) Intra-operative view, after tumor excision. (c) Immediate post-operative view after flap inset. (d) Four month post-operative view.

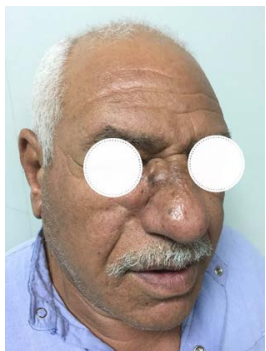

(a)

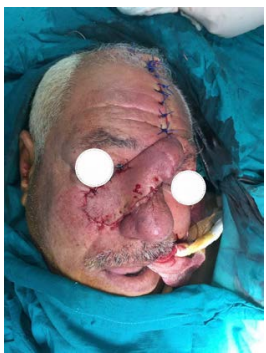

(c)

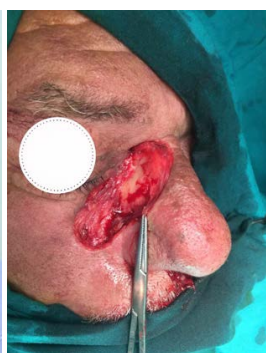

(b)

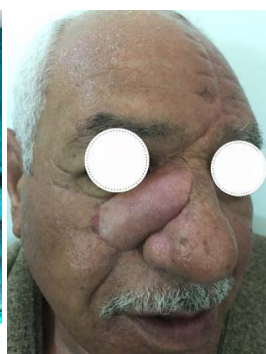

(d)

Case 2. A 70-year-old male with basal cell carcinoma of the right upper medial check and lateral nasal wall and part of the upper dorsum. (a) Pre-operative Antero-lateral view of the face showing the tumor. (b) Intra-operative view, after tumor excision and flap elevation. (c) Immediate post-operative view after flap inset. (d) Eight month post-operative view. 


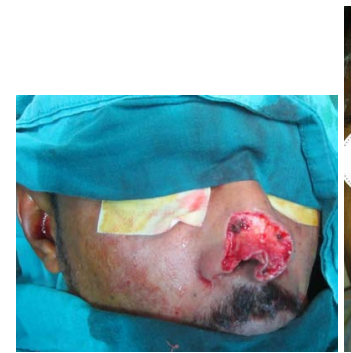

(a)

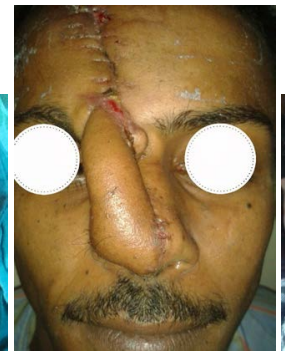

(b)

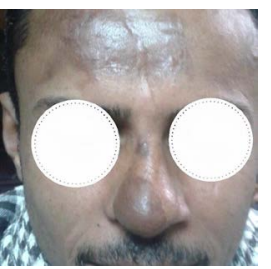

(c)

Case 3. A 33-year-old male with trauma to the nose with loss of part of the tip and right ala. (a) Intra-operative view, after wound debridement. (b) One week post-operative view. (c) One year post-operative view.

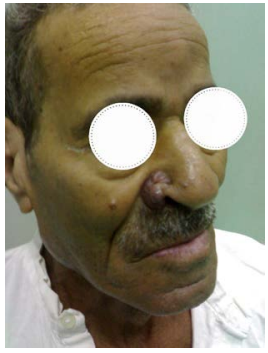

(a)

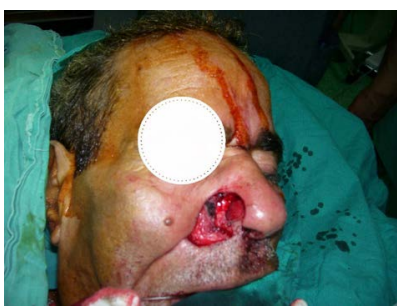

(b)

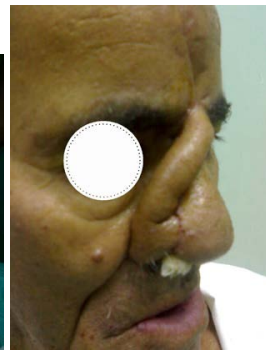

(c)

Case 4. A 66-year-old male with basal cell carcinoma of the right medial check and lateral nasal wall and part of the right ala. (a) Pre-operative Antero-lateral view of the face showing the tumor. (b) Intra-operative view, after tumor excision. (c) Two weeks post-operative view. 